\title{
Knockdown of TAZ modifies triple-negative breast cancer cell sensitivity to EGFR inhibitors by regulating YAP expression
}

\author{
LIWEN GUO $^{1,2}$, JIAPING ZHENG ${ }^{2}$, JING ZHANG ${ }^{1}$, HAOHAO WANG $^{1}$, \\ GUOLIANG SHAO ${ }^{2}$ and LISONG TENG ${ }^{1}$
}

\author{
${ }^{1}$ Department of Surgical Oncology, The First Affiliated Hospital, School of Medicine, \\ Zhejiang University, Hangzhou, Zhejiang 310003; ${ }^{2}$ Department of Intervention Therapy, \\ Zhejiang Cancer Hospital, Hangzhou, Zhejiang 310022, P.R. China
}

Received January 12, 2016; Accepted February 18, 2016

DOI: $10.3892 /$ or.2016.4875

\begin{abstract}
Triple-negative breast cancer (TNBC) constitutes $\sim 10-15 \%$ of breast cancer patients and represents an aggressive subtype with poor overall prognosis. TNBC is an important clinical challenge because it does not respond well to endocrine therapy and have a higher rate of early recurrence and distant metastasis following chemotherapy. Although it has been reported that the epidermal growth factor receptor (EGFR) was overexpressed in $\sim 80 \%$ of TNBC, anti-EGFR therapy showed limited clinical benefit according to phase II studies. In this study, we first observed that knockdown of the transcriptional coactivator with PDZ-binding domain (TAZ) gene can regulate the sensitivity of TNBC cell lines to EGFR inhibitors (EGFRI) in a cell context-depended manner. Furthermore, in certain breast cancer cell lines the YES-associated protein, paralog of TAZ (YAP) expression can be upregulated by TAZ inhibition which leads to EGFRI resistance. These results suggest a specific inhibitor to TAZ/YAP combined with anti-EGFR therapy may prove effective and provide a reason why targeting EGFR showed limited clinical benefit in TNBC treatment.
\end{abstract}

\section{Introduction}

Breast cancer is the most frequently diagnosed cancer in women around the world (1). Although the mortality has declined over the two decades mainly due to the deeper understanding of its biology and advances in management approaches, it is still the most life-threatening cancer in women, and, even worse,

Correspondence to: Professor Guoliang Shao, Department of Intervention Therapy, Zhejiang Cancer Hospital, 38 Guangji Road, Hangzhou, Zhejiang 310022, P.R. China

E-mail: shaoguoliang2008@126.com

Professor Lisong Teng, Department of Surgical Oncology, The First Affiliated Hospital, School of Medicine, Zhejiang University, 79 Qingchun Road, Hangzhou, Zhejiang 310003, P.R. China

E-mail: lsteng@zju.edu.cn; guoliwen2008@126.com

Key words: transcriptional coactivator with PDZ-binding domain, YES-associated protein, paralog of TAZ, Hippo, breast cancer, epidermal growth factor receptor the incidence rate is increasing gradually (2). Through gene expression profiling, several intrinsic breast cancer subtypes have been identified. Triple-negative breast cancer (TNBC), one of the subtypes, accounting for $10-15 \%$ of invasive breast cancers, is characterized by the absence of estrogen receptor and progesterone receptor and no overexpression of human epidermal growth factor receptor 2 (HER2) (3). Therefore, patients with TNBC cannot be treated with endocrine therapy or therapies targeted to HER2. As a group, they have a worse prognosis and tend to relapse early compared with other subtypes of breast cancers (4). Hence, there is a compelling need to find more effective treatments. It has been reported that the overexpression of epidermal growth factor receptor (EGFR) was seen in $\sim 80 \%$ of TNBC $(5,6)$. This discovery led to the investigation of the EGFR inhibitors (EGFRI). However, both the anti-EGFR monoclonal antibody cetuximab and the small molecular tyrosine kinase inhibitors (TKIs) gefitinib and erlotinib seem to be ineffective according to phase II studies (7-9). Thus, more studies are needed to answer the question of why EGFR inhibitors failed in treatment of those EGFR overexpressed breast cancers.

TAZ (transcriptional coactivator with PDZ-binding domain; also known as WWTR1) and its paralog YAP (YES associated protein) are the two main downstream effectors of the Hippo signaling pathway, which plays a major role in organ size control, cell differentiation, and tumorigenesis across species (10). TAZ is preferentially overexpressed in highly invasive breast cancer cells, most of which belong to TNBC cell lines (11). In addition, it has been reported that overexpression of TAZ induced the activation of EGFR signaling, and one of the EGFR ligands, amphiregulin (AREG), is a target of TAZ. AREG functions in a non-cell-autonomous manner to mediate EGF-independent growth and malignant behavior of mammary epithelial cells (12). These studies suggest that the high expression of TAZ may be one of the reasons that TNBC was not sensitive to EGFR inhibitors.

In this study, we successfully established two types of TNBC cell lines with TAZ stably silenced. For the first time, we observed TAZ gene silencing modified the drug sensitivity of breast cancer cells to EGFRI in a cell context-depended manner. In addition, also for the first time, we found YAP expression could be upregulated both at mRNA and protein 
levels by TAZ inhibition in certain breast cancer cell line, which leads to the EGFRI resistance. These findings indicate that TAZ/YAP inhibition can significantly improve EGFRI efficacy, which may pave the way for TNBC therapy.

\section{Materials and methods}

Cell culture and antibodies. Human breast cancer cell lines (MCF-7, MDA-MB-231, MDA-MB-468, and BT-549) were obtained from American Type Culture Collection. These original cells were routinely cultured at $37^{\circ} \mathrm{C}$ in the presence of $5 \% \mathrm{CO}_{2}$ in RPMI-1640 supplemented with $10 \%$ fetal bovine serum (FBS, Hyclone). Cells in the exponential growth phase were used for all experiments. The primary antibodies, anti-YAP, anti-TAZ/YAP, and anti-GAPDH, were purchased from Cell Signaling Technology Inc., and HRP-conjugated secondary antibodies against rabbit from GE Amersham.

Cell lysates preparation and western blot analysis. Briefly, cells were washed twice with phosphate-buffered saline (PBS) and then lysed on ice in RIPA buffer (50 mM Tris- $\mathrm{HCl} \mathrm{pH} \mathrm{7.4,}$ $1 \%$ Nonidet P-40, $0.5 \%$ sodium deoxycholate, $150 \mathrm{mM} \mathrm{NaCl}$, $0.02 \%$ sodium azide, and $0.1 \%$ SDS) containing protease and phosphatase inhibitors (Sigma-Aldrich, St. Louis, MO, USA) for $15 \mathrm{~min}$ and cleared of debris by centrifugation at $12,000 \mathrm{rpm}$ for $15 \mathrm{~min}$ at $4^{\circ} \mathrm{C}$. After boiling with an equal volume of $2 \mathrm{X}$ SDS loading buffer for $5 \mathrm{~min}$, cell lysates were electrophoresed with 10\% SDS-PAGE and blotted to PVDF membranes (Millipore). The membranes were blotted with $5 \%$ non-fat milk in TBS-T (10 mmol/1 Tris-HCl pH 7.5,0.5 mol/1 $\mathrm{NaCl}$, and $0.05 \% \mathrm{w} / \mathrm{v}$ Tween-20) buffer at room temperature for $1 \mathrm{~h}$, and then incubated with primary antibodies overnight at $4^{\circ} \mathrm{C}$. The membranes were washed and then incubated with suitable peroxidase conjugated secondary antibodies for $1 \mathrm{~h}$ at room temperature. After washing three times with TBS-T, antibody binding was visualized using chemiluminescence detection system as described by the manufacturer (Millipore). Molecular weights of the immunoreactive proteins were estimated based on PageRuler Prestained Protein ladder (MBI, Fermentas). Experiments were repeated at least three times.

$R N A$ purification and quantitative reverse transcriptase$P C R$ (RT-qPCR). Total RNA was extracted using TRIzol reagent according to the protocol provided by the manufacturer (Invitrogen). RNA concentrations were quantified by NanoDrop 2000 (Nanodrop). Reverse transcription reaction was performed using $2 \mu \mathrm{g}$ of total RNA with Reverse Transcription System (Promega). The mRNA levels of TAZ and YAP were analyzed using SYBR-Green qPCR Master Mix kit (Promega) in ABI PRISM 7500 fast Sequence Detection System (Applied Biosystems). The real-time qPCR reaction was carried out in triplicate for each sample. The GAPDH gene was used as an endogenous control for normalization and the mRNA levels of TAZ and YAP were determined using the $2^{-\Delta \Delta C t}$ methods (13). Specific primer pairs are listed in Table I.

siRNA transfection and lentivirus infection. The small interfering RNAs (siRNAs) against TAZ and YAP were designed and synthesized by GenePharma Inc. (GenePharma) and transfection was done with Lipofectamine ${ }^{\mathrm{TM}}$ RNAiMAX
Table I. Sequences of primers for real-time qPCR.

\begin{tabular}{ll}
\hline Name & \multicolumn{1}{c}{ Primer sequences } \\
\hline GAPDH & F:TGATGACATCAAGAAGGTGGTGAAG \\
& R: TCCTTGGAGGCCATGTGGGCCAT \\
TAZ & F: CAGCAATGTGGATGAGATGG \\
& R: AAGGAGGGAGCACGAGTCA \\
YAP & F: GGAACACTGGAAGGAGATGG \\
& R: AGCAATGGACAAGGAAGAGC \\
\hline
\end{tabular}

F, Forward. R, reverse.

(Life Technologies) regent according to the manufacturer's protocol. The regions of the TAZ mRNA (GenBank accession no. NM_001168278) and YAP mRNA (GenBank accession no. NM_006106) were selected as the RNAi target sites. The RNA interfering sequences were 5'-CCGUUUCCCUGAUU UCCUUTT-3' (sense) for si-TAZ and 5'-GGUGAUACUAUCA ACCAAATT-3' (sense) for si-YAP. BLAST analysis shows no homology of the siRNA sequences to any other sequences in the Human Genome Database. Scrambled siRNA as negative control was also obtained from GenePharma.

To establish stable TAZ knockdown cells (BT-549/sh-TAZ; MDA-MB-231/sh-TAZ), biologically active short hairpin RNA (shRNA) was subcloned into lentiviral vector hU6-MCSUbiquitin-EGFP-IRES-puromycin (GV248, Genechem), which carried the transgene for green fluorescent protein (GFP), and used to infect BT-549 and MDA-MB-231 cells. The shRNA target sequence was the same target sequence of the si-TAZ mentioned above. Breast cancer cells were incubated with viral supernatants for $24 \mathrm{~h}$ and then returned to normal growth medium. For confirmation of downregulation of TAZ gene, after $72 \mathrm{~h}$ cells were harvested and analyzed for reduction of TAZ expression by real-time RT-qPCR and western blot analysis.

Flow cytometry assay. Apoptosis induction in siRNA treated cells was assayed by the detection of membrane externalization of phosphatidylserine using an Annexin V-fluorescein isothiocyanate (FITC)/propidium iodide (PI) apoptosis assay kit (KeyGen Biotech). At $72 \mathrm{~h}$ after transfection, cells were harvested and washed with ice-cold PBS twice and resuspended in $500 \mu \mathrm{l}$ of binding buffer, $5 \mu \mathrm{l}$ Annexin V-FITC and $5 \mu \mathrm{l}$ PI were added, and then cells were incubated for $15 \mathrm{~min}$ in the dark. Finally, the cells were analyzed within $1 \mathrm{~h}$ by flow cytometry.

Cell proliferation assay. Cell proliferation was investigated by colorimetric assay using 3-(4, 5-dimethylthiazol-2-yl)-2, 5-diphenyltetrazolium bromide (MTT). In brief, breast cancer cells were seeded in a 96-well flat-bottomed plate at $5 \times 10^{3}$ cells/ well in triplicate. At each experimental point, $0.5 \mathrm{mg} / \mathrm{ml} \mathrm{MTT}$ (Sigma-Aldrich) was added into the medium and cells were cultured for additional $4 \mathrm{~h}$. Afterwards, the supernatant was removed and the formazan crystals were dissolved in $150 \mathrm{ml}$ dimethyl sulfoxide (DMSO) at room temperature for $15 \mathrm{~min}$. Absorbance of the solution was then measured at $490 \mathrm{~nm}$ 

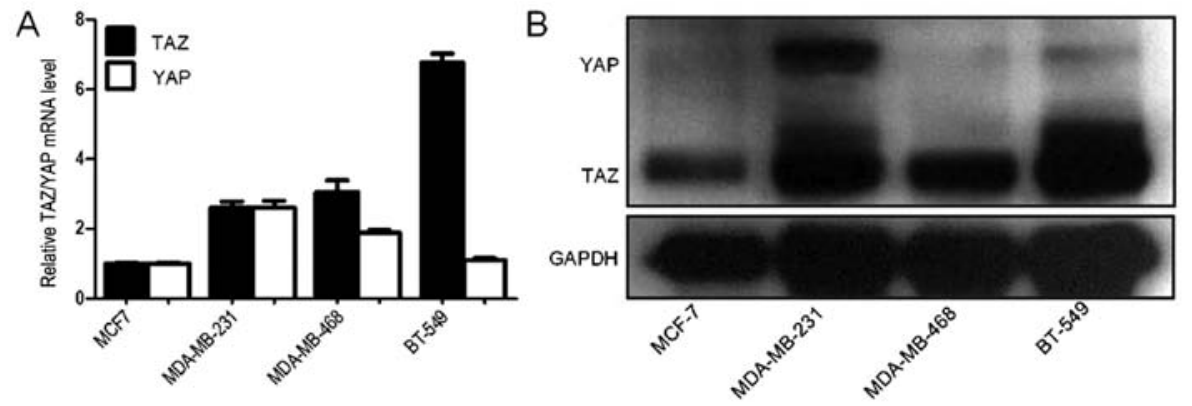

Figure 1. TAZ and YAP expression in four breast cancer cell lines. (A) Expression of TAZ and YAP mRNA was examined by RT-qPCR. (B) Lysates derived from the breast cancer cell lines were analyzed by western blotting using anti-TAZ antibodies; these anti-TAZ antibodies also reacted well with YAP. The levels of GAPDH as detected by anti-GAPDH antibodies were used as loading controls.
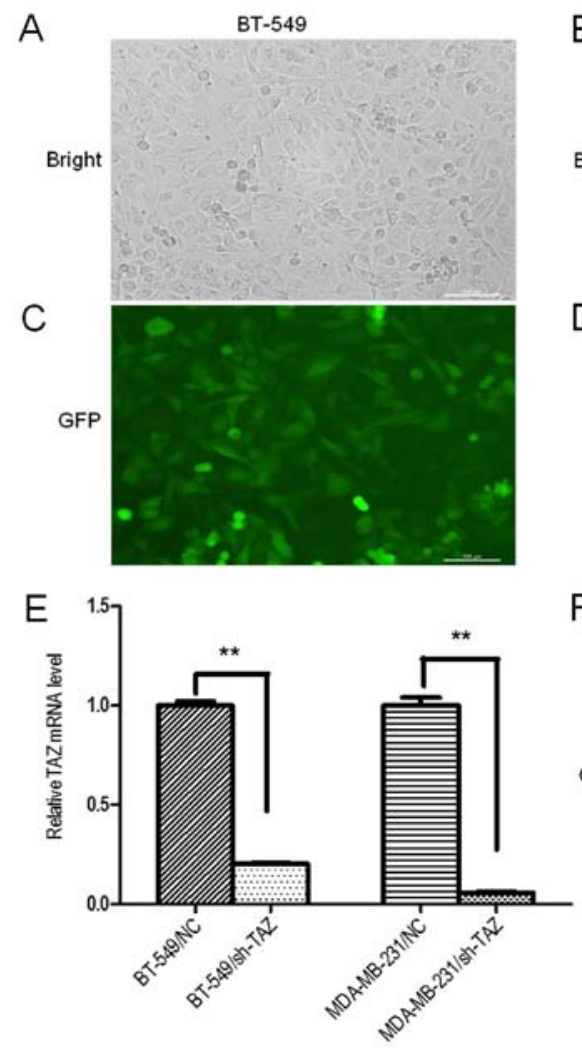

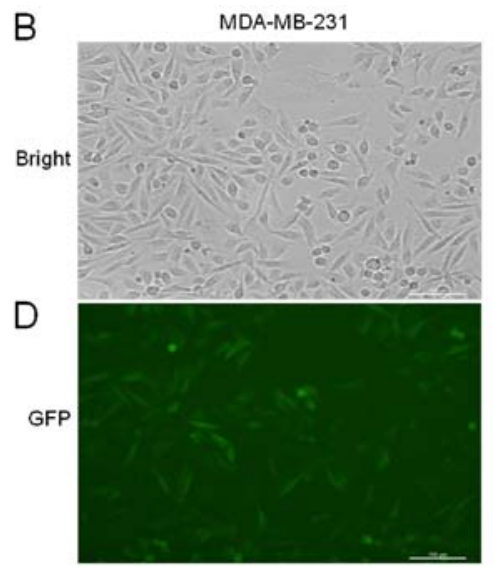

$\mathrm{F}$

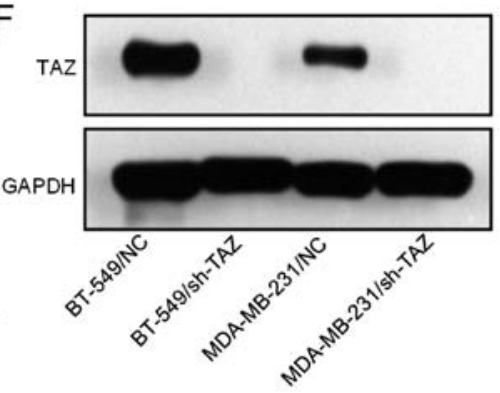

Figure 2. TAZ shRNA lentivirus-silenced TAZ expression in the BT-549 cells and MDA-MB-231 cells. BT-549 (A and C) and MDA-MB-231 cells (B and D) were successfully infected with TAZ shRNA lentivirus at MOI 30 at $72 \mathrm{~h}$; a fluorescence microscope system was used for observing the expression of green fluorescence protein (GFP). Light micrograph (magnification, x400) (upper panel); fluorescent micrograph (magnification, x400) (lower panel). (E) TAZ mRNA expression decreased significantly in $72 \mathrm{~h}$ post-infection with TAZ shRNA detected by real-time RT-PCR. The data were normalized to the negative control. (F) TAZ protein expression was significantly reduced at $72 \mathrm{~h}$ post-infection by immunoblotting.

wavelength using an ELx800 Absorbance Microplate Reader (Biotek). The experiments were performed independently in triplicate.

Chemosensitivity assay. EGFR inhibitor, gefitinib or AG-1478 (Selleckchem) was initially dissolved in DMSO, which was diluted with fresh medium immediately before each experiment. Briefly, BT-549/sh-TAZ or MDA-MB-231/sh-TAZ cells were seeded in a 96 -well plate at $5 \times 10^{3}$ cells/well in triplicate and incubated for $24 \mathrm{~h}$. Then the medium was moved and replaced with the fresh medium containing the drugs with different concentrations. After incubation for another $48 \mathrm{~h}$, the cell viability was examined by the MTT assay.
Colony formation assay. Log-phase cells were seeded in triplicate onto 6-well plates with $2 \mathrm{ml}$ of complete media (500 cells/ well) and incubated at $37^{\circ} \mathrm{C}$ in a humidified incubator. Every week the medium was replaced with fresh medium. After 3 weeks, the colonies were fixed with $100 \%$ methanol, stained with $0.1 \%$ crystal violet, and washed with PBS. The numbers of colonies were counted using a light microscope.

Statistical analysis. The results were expressed as mean \pm standard deviation. Statistical significance was assessed by Student's t-test or one-way ANOVA followed by Bonferroni multiple comparison post-tests. Statistical analyzes were performed using GraphPad Prism v.5.0 package. Differences 

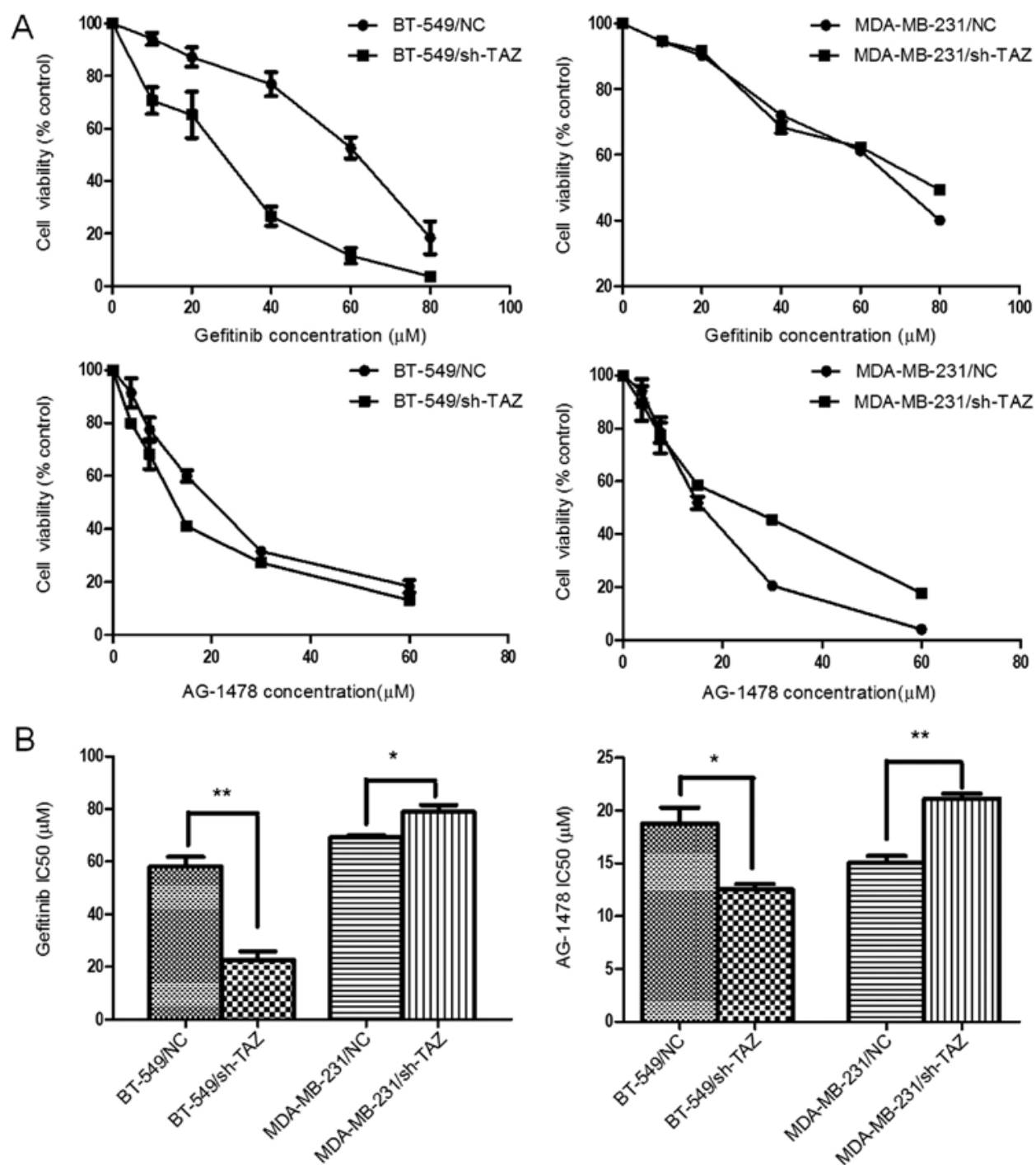

Figure 3. Inhibition of gefitinib or AG-1478 on EGFRI sensitivity in TAZ expression modified BT-549 cells and MDA-MB-231 cells. (A) Inhibition curves of gefitinib and AG-1478 at six different drug concentrations. (B) Knockdown of TAZ in BT-549 cells showed higher sensitivity to gefitinib and AG-1478 as evidenced by lower $\mathrm{IC}_{50}$, but TAZ knockdown induced gefitinib and AG-1478 resistance in MDA-MB-231 cells.

were considered statistically significant at a level of $\mathrm{P}<0.05$ (shown as ${ }^{*} \mathrm{P}<0.05 ;{ }^{* *} \mathrm{P}<0.01$ in the figures).

\section{Results}

TAZ expression was upregulated in triple-negative breast cancer cells. The expression of TAZ and YAP was examined by western blotting and RT-qPCR in 4 human breast cancer cell lines. TAZ is preferentially overexpressed in TNBC cells (BT-549, MDA-MB-468, and MDA-MB-231); however, high expression level of YAP was only seen in MDA-MB-231 cells (Fig. 1). These results are consistent with a previous study (14). TAZ is comparably highly expressed in TNBC cells suggesting that it may be correlated with certain characteristics of TNBC (15).

sh-TAZ regulates breast cancer cell sensitivity to EGFRI gefitinib and $A G-1478$. In order to establish breast cancer cells with TAZ stably silenced, we successfully constructed a lentivirus vector harboring shRNA against TAZ. We chose
TAZ overexpressing cells, BT-549 and MDA-MB-231, for TAZ knockdown. The knockdown efficiency was evaluated using western blotting and RT-qPCR. The results disclosed that the best knockdown effect was with shRNA at multiplicity of infection (MOI) 30 both in BT-549 and MDA-MB-231 cells. After $72 \mathrm{~h}$ post-transfection, $>90 \%$ of the survived cells were GFP-positive (Fig. 2A-D). RT-qPCR analyses showed that TAZ mRNA levels were significantly reduced when compared with corresponding negative control transfection (Fig. 2E). To correlate the decreases in TAZ mRNA expression with TAZ protein levels, western blot analysis was performed at $72 \mathrm{~h}$ after shRNA silencing and showed that TAZ protein levels were also reduced, thereby confirming efficient knockdown (Fig. 2F).

To investigate the effect of sh-TAZ on EGFRI sensitivity in the breast cancer cells, we treated TAZ shRNA or negative control (NC) shRNA transfected cells with gefitinib or AG-1478 separately and the cell viability curves are shown in Fig. 3A. The silencing of TAZ expression in BT-549 cells resulted in strikingly higher cell growth inhibition at 

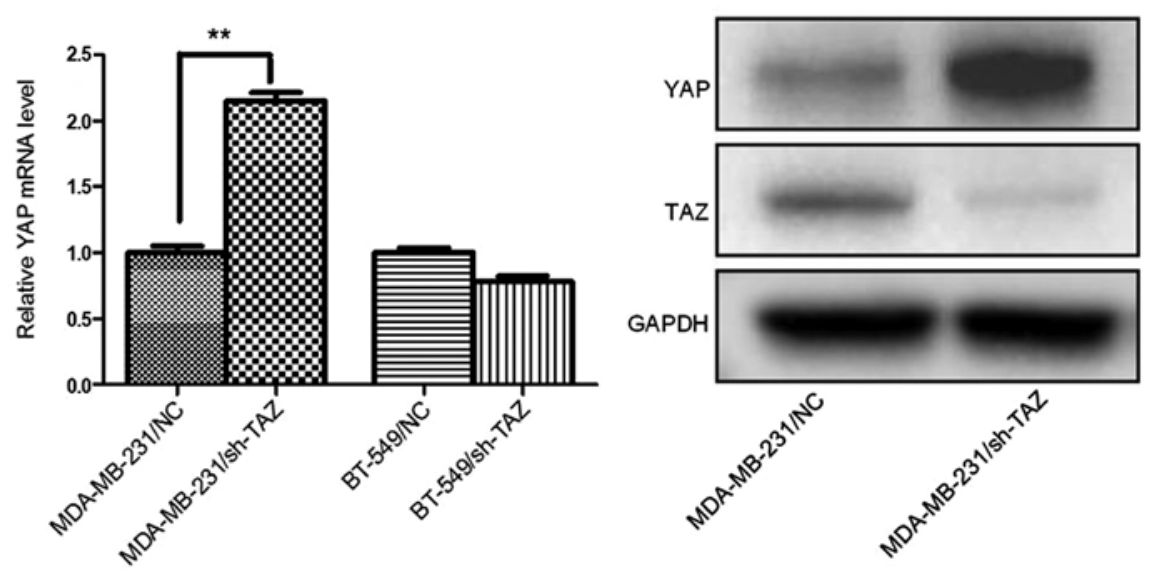

Figure 4. Knockdown of TAZ in MDA-MB-231 cells induces upregulation of YAP expression both at mRNA and protein levels, but not in BT-549 cells.
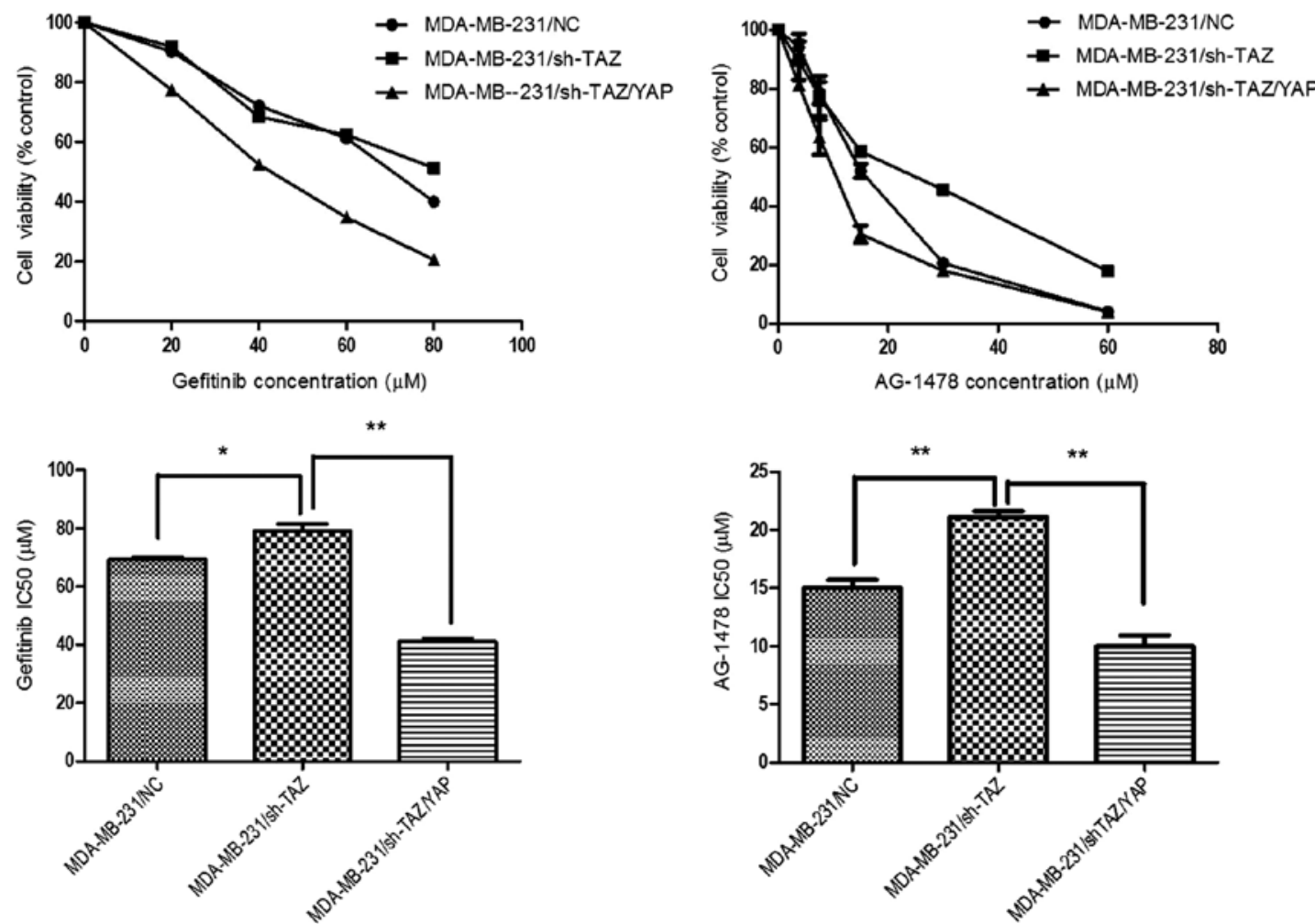

Figure 5. Co-knockdown of TAZ and YAP reverses gefitinib and AG-1478 resistance in MDA-MB-231/sh-TAZ cells.

different drug concentrations, with the $\mathrm{IC}_{50}$ of gefitinib being $22.65 \pm 3.28 \mu \mathrm{M}$ in BT-549/sh-TAZ cells, significantly lower than $58.19 \pm 3.58 \mu \mathrm{M}$ in $\mathrm{BT}-549 / \mathrm{NC}$ cells $(\mathrm{P}=0.002)$. The same trend was found in $\mathrm{AG}-1478$ treatment, with the $\mathrm{IC}_{50}$ reduced from $18.76 \pm 1.52$ to $12.52 \pm 0.53 \mu \mathrm{M}(\mathrm{P}=0.018)$. In contrast, shRNA-TAZ in MDA-MB-231 cells led to EGFRI resistance, with the $\mathrm{IC}_{50}$ values of gefitinib and AG-1478 in MDA-MB-231/NC cells being 69.27 \pm 0.86 and $15.02 \pm 0.68 \mu \mathrm{M}$, respectively, significantly lower than $79.01 \pm 2.54(\mathrm{P}=0.022)$ and $21.14 \pm 0.49 \mu \mathrm{M}(\mathrm{P}=0.002)$ in MDA-MB-231/sh-TAZ cells (Fig. 3B). These results suggest that TAZ inhibition by lentiviral shRNA resulted in enhancement in chemosensitivity of EGFRI in BT-549 cells, but was diminished in MDA-MB-231 cells.
The sh-TAZ led EGFRI resistance in MDA-MB-231 cells was mediated by upregulation of YAP expression. To understand the underlying mechanism of the effect of sh-TAZ on EGFRI sensitivity in the breast cancer cells, we further analyzed the expression change of YAP before and after TAZ knockdown. As shown in Fig. 1, YAP expression in MDA-MB-231 cells was higher than that in MCF-7, but was slight or absent in BT-549 cells. In addition, after TAZ knockdown, the expression of YAP in MDA-MB-231/sh-TAZ cells was markedly elevated both in mRNA and protein levels, which was not seen in BT-549/sh-TAZ cells (Fig. 4).

In order to study the relationship between YAP expression and EGFRI resistance, we further knocked down YAP in MDA-MB-231/sh-TAZ cells by YAP specific siRNA, and inves- 

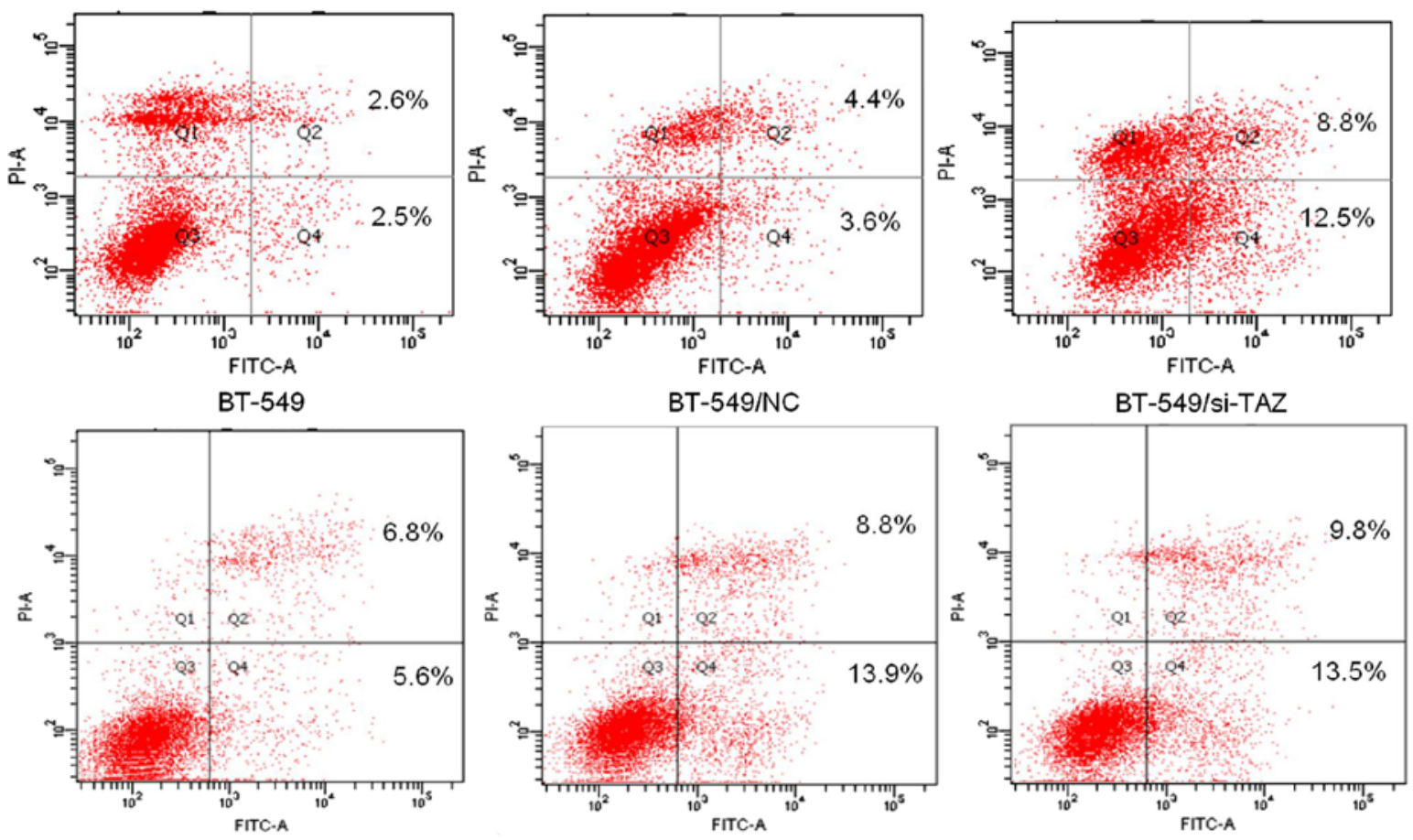

MDA-MB-231

MDA-MB-231/NC

MDA-MB-231/si-TAZ

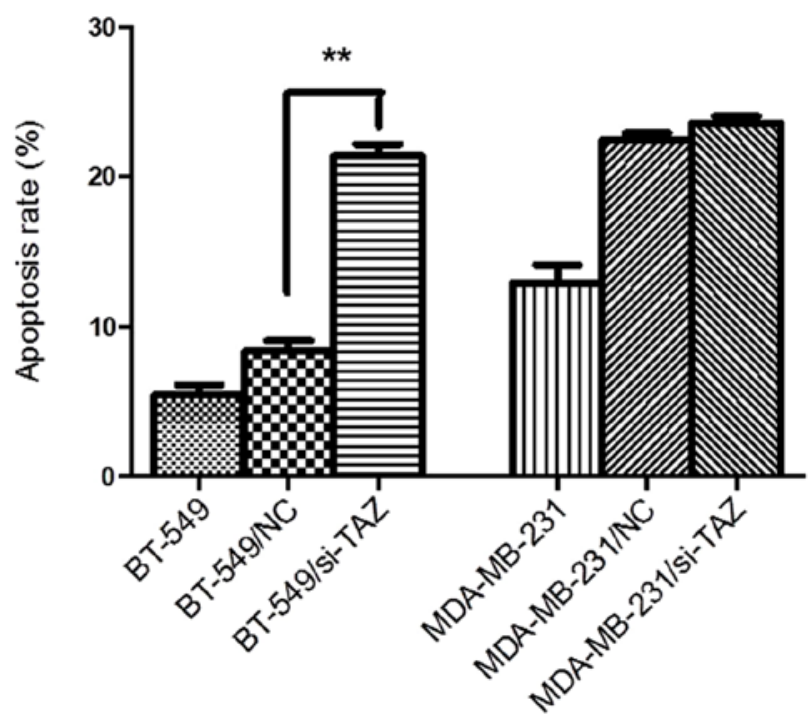

Figure 6. Effects of TAZ knockdown on apoptosis of BT-549 and MDA-MB-231 cells. Apoptosis was assessed after transfection with TAZ specific siRNA for $48 \mathrm{~h}$. Flow cytometry revealed that knockdown of TAZ by specific siRNA induced apoptosis in BT-549 cells, but not in MDA-MB-231 cells. ${ }^{* *} \mathrm{P}<0.01$.

tigated the changes of $\mathrm{IC}_{50}$. The results show that $\mathrm{IC}_{50}$ values of gefitinib and AG-1478 were $79.01 \pm 2.54$ and $21.14 \pm 0.49 \mu \mathrm{M}$ for MDA-MB-231/sh-TAZ cells, respectively, but $\mathrm{IC}_{50}$ values in TAZ/YAP co-knockdown MDA-MB-231 cells declined to $41.02 \pm 1.26(\mathrm{P}<0.01)$ and $9.98 \pm 0.96 \mu \mathrm{M}(\mathrm{P}<0.01)$, respectively (Fig. 5). These results suggest that compared with the MDA-MB-231/sh-TAZ cells, the TAZ/YAP co-knockdown MDA-MB-231 cells restored the EGFRI sensitivity.

TAZ inhibition affects apoptosis and proliferation of breast cancer cells. To determine whether inhibition of TAZ affected apoptosis and proliferation in breast cancer cells, we performed flow cytometry, cell proliferation curve, and colony forming assays. Flow cytometry showed the cellular apoptosis was significantly increased in the BT-549 cells transfected with TAZ specific siRNA (BT-549/si-TAZ) compared with control, while no significant change was seen in MDA-MB-231/si-TAZ cells, suggesting that si-TAZ induced spontaneous apoptosis in BT-549 cells (Fig. 6). Similarly, cell proliferation curves by MTT assay show that silencing of TAZ gene substantially affected BT-549 cells on proliferation compared with control; however, silencing of TAZ gene slightly promoted cell proliferation in MDA-MB-231 cells (Fig. 7). The colony forming efficiency of the transfected cells was investigated by colony forming assay, and the results show that TAZ-shRNA transfected BT-549 cells had significantly fewer colonies than control, while, on the contrary, MDA-MB-231/sh-TAZ cells showed more colonies than control (Fig. 7). 


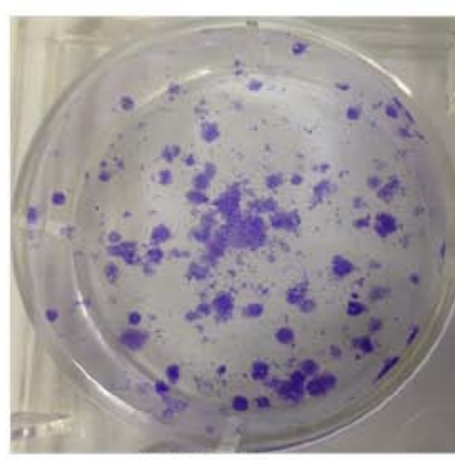

BT-549/NC

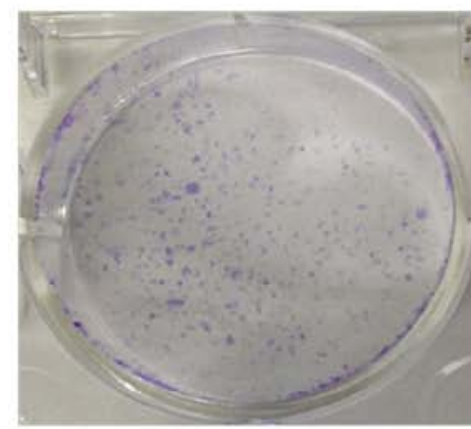

MDA-MB-231/NC

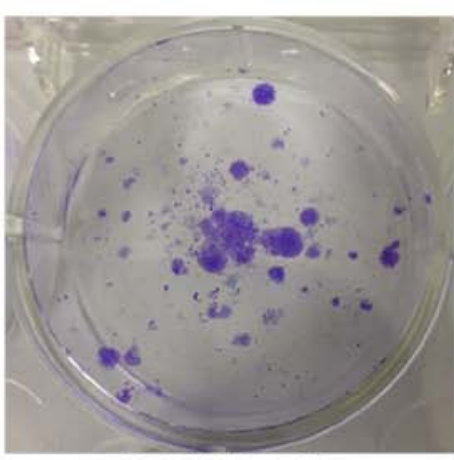

BT-549/sh-TAZ

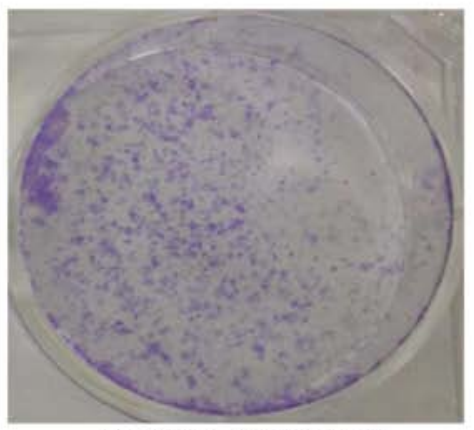

MDA-MB-231/sh-TAZ
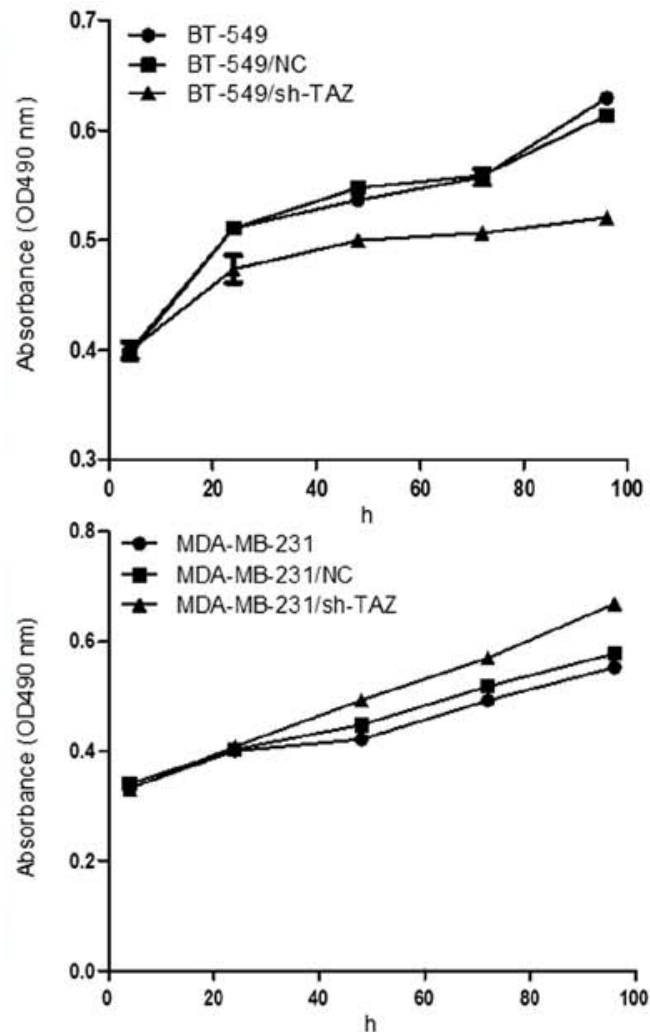

Figure 7. Effects of TAZ knockdown on proliferation of BT-549 and MDA-MB-231 cells The proliferation curves by MTT assay shows the growth rate of BT-549/sh-TAZ cells was inhibited, while that of MDA-MB-231/sh-TAZ cells was increased, compared with negative control and parent cells. Colony forming assay showed that TAZ-shRNA transfected BT-549 cells formed significantly fewer colonies than control, while the MDA-MB-231/sh-TAZ cells showed more colonies than control.

\section{Discussion}

The Hippo signaling pathway is a newly discovered and evolutionally conserved signal cascade, which plays a pivotal role in regulating organ size, stem cell pluripotency, and tumorigenesis from Drosophila to mammals (15). Mechanically, when the main downstream effectors TAZ or YAP translocated into the nucleus, they will act as transcription coactivators to promote proliferation-associated gene expression (16). Despite highly conserved sequence and domain organization, TAZ and YAP have their own specific transcription factor partners, and some researchers believe they can hardly compensate each other (17).

Indeed, it has been reported that TAZ expression level was increased in a broad range of different human cancers, such as colorectal, breast, and lung cancers. Moreover, the higher TAZ protein level is always associated with poorly differentiated tumors and shorter patient overall survival (18). In vitro experiments demonstrate that downregulation of TAZ expression not only reduced cancer cell migration and invasion, but also inhibited tumorigenesis in nude mice, while upregulation was able to induce cell malignant transformation (11). Therefore, TAZ is proposed as an oncogene and a potentially attractive therapeutic target for cancer treatment (19).

We have noted that TAZ can induce growth factors to promote independent proliferation of breast cancer cells through activation of its transcription target EGFR ligand AREG, and expression of TAZ and EGFR is positively corre- lated with the invasiveness of breast cancer cell lines (12). These observations implicate the potential benefit of TAZ knockdown on EGFR targeted therapy. However, no published studies exist focused on this issue in the PubMed, although TAZ mediated Taxol resistance in breast cancer cells have been reported (20).

In this study, we investigated the therapeutic effects of EGFRI on human breast cancer cells overexpressing TAZ. Gefitinib, an EGFRI, has been shown to be highly effective in clinical treatment of certain pathological types of lung cancer, and AG-1478, which is a highly selective EGFRI, has almost no activity on HER2, platelet-derived growth factor receptor (PDGFR), tyrosine kinase receptor (Trk), Bcr-abl or insulin receptor (InsR). Interestingly, BT-549 and MDA-MB-231 cell lines showed different responses to EGFRI treatment when TAZ was silenced, with an increase in chemosensitivity of BT-549/sh-TAZ cells to EGFRI and a decrease in MDA-MB-231/sh-TAZ cells.

Further research found that BT-549 cells expressed a high level of TAZ, but a low level of YAP, and the level of YAP did not increase after TAZ knockdown. By contrast, MDA-MB-231 cells expressed both TAZ and YAP, and the level of YAP significantly increased after TAZ knockdown. In order to identify whether the EGFRI resistance of MDA-MB-231/sh-TAZ cells was caused by increased expression of YAP, we further knocked down the YAP in MDA-MB-231/sh-TAZ cells, and found that the resistance to EGFRI in MDA-MB-231/sh-TAZ cells was reversed. 
Previous studies have shown that TAZ may compensate for the loss of YAP functions. Huang et al reported that knockdown of YAP significantly increased EGFRI erlotinib sensitivity in ovarian cancer cell lines that express little or no TAZ (21). In addition, knockdown of YAP in ovarian cancer cell lines that express both YAP and TAZ only led to a very moderate effect on cancer cell growth or drug sensitivity $(22,23)$. Here, we have shown that knockdown of TAZ in breast cancer cell lines that express little or no YAP, such as BT-549 cells, increased EGFRI sensitivity; however, for breast cancer cell lines that express both YAP and TAZ, such as MDA-MB-231 cells, knockdown of TAZ may not help improve sensitivity of EGFRI treatment. These findings indicate YAP may also compensate for the loss of TAZ functions. Therefore, simultaneous inhibition of the functions of TAZ and YAP is needed in some cases.

In conclusion, this study highlights the potential for TAZ to be a therapeutic target in breast cancers, as reducing TAZ levels can partially revert resistance to EGFR inhibitors. In addition, for the first time, we found upregulation of YAP could be induced by TAZ inhibition in a certain breast cancer cell line, which leads to EGFRI resistance. For patients with high expression of both TAZ and YAP, anti-YAP drugs need to be added. Therefore, we propose to develop new therapeutic agents that can simultaneously target TAZ and YAP. We believe that a specific inhibitor to TAZ/YAP combined with anti-EGFR therapy may improve the therapeutic efficacy in TNBC treatment.

\section{Acknowledgements}

This study was supported in part by the National Natural Science Foundation of China for the youth (no. 81301809), and the Cultivation of High-level Innovation Health Talents of Zhejiang (grant no. 2012-241).

\section{References}

1. Boyle P and Howell A: The globalisation of breast cancer. Breast Cancer Res 12 (Suppl 4): S7, 2010.

2. Youlden DR, Cramb SM, Yip CH and Baade PD: Incidence and mortality of female breast cancer in the Asia-Pacific region. Cancer Biol Med 11: 101-115, 2014.

3. Brenton JD, Carey LA, Ahmed AA and Caldas C: Molecular classification and molecular forecasting of breast cancer: Ready for clinical application? J Clin Oncol 23: 7350-7360, 2005.

4. Criscitiello C, Azim HA Jr, Schouten PC, Linn SC and Sotiriou C: Understanding the biology of triple-negative breast cancer. Ann Oncol 23 (Suppl 6): vi13-vi18, 2012.

5. De Laurentiis M, Cianniello D, Caputo R, Stanzione B, Arpino G, Cinieri S, Lorusso V and De Placido S: Treatment of triple negative breast cancer (TNBC): Current options and future perspectives. Cancer Treat Rev 36 (Suppl 3): S80-S86, 2010.

6. Corkery B, Crown J, Clynes M and O'Donovan N: Epidermal growth factor receptor as a potential therapeutic target in triplenegative breast cancer. Ann Oncol 20: 862-867, 2009.
7. Bernsdorf M, Ingvar C, Jörgensen L, Tuxen MK, Jakobsen EH, Saetersdal A, Kimper-Karl ML, Kroman N, Balslev E and Ejlertsen B: Effect of adding gefitinib to neoadjuvant chemotherapy in estrogen receptor negative early breast cancer in a randomized phase II trial. Breast Cancer Res Treat 126: 463-470, 2011.

8. Carey LA, Rugo HS, Marcom PK, Mayer EL, Esteva FJ, Ma CX, Liu MC, Storniolo AM, Rimawi MF, Forero-Torres A, et al: TBCRC 001: Randomized phase II study of cetuximab in combination with carboplatin in stage IV triple-negative breast cancer. J Clin Oncol 30: 2615-2623, 2012.

9. Dickler MN, Cobleigh MA, Miller KD, Klein PM and Winer EP: Efficacy and safety of erlotinib in patients with locally advanced or metastatic breast cancer. Breast Cancer Res Treat 115: 115-121, 2009.

10. Hong W and Guan KL: The YAP and TAZ transcription co-activators: Key downstream effectors of the mammalian Hippo pathway. Semin Cell Dev Biol 23: 785-793, 2012.

11. Chan SW, Lim CJ, Guo K, Ng CP, Lee I, Hunziker W, Zeng Q and Hong W: A role for TAZ in migration, invasion, and tumorigenesis of breast cancer cells. Cancer Res 68: 25922598, 2008.

12. Yang N, Morrison CD, Liu P, Miecznikowski J, Bshara W, Han S, Zhu Q, Omilian AR, Li X and Zhang J: TAZ induces growth factor-independent proliferation through activation of EGFR ligand amphiregulin. Cell Cycle 11: 2922-2930, 2012.

13. Livak KJ and Schmittgen TD: Analysis of relative gene expression data using real-time quantitative PCR and the 2(-Delta Delta C(T)) method. Methods 25: 402-408, 2001.

14. Cordenonsi M, Zanconato F, Azzolin L, Forcato M, Rosato A Frasson C, Inui M, Montagner M, Parenti AR, Poletti A, et al: The Hippo transducer TAZ confers cancer stem cell-related traits on breast cancer cells. Cell 147: 759-772, 2011.

15. Pan D: The hippo signaling pathway in development and cancer. Dev Cell 19: 491-505, 2010.

16. Piccolo S, Cordenonsi M and Dupont S: Molecular pathways: YAP and TAZ take center stage in organ growth and tumorigenesis. Clin Cancer Res 19: 4925-4930, 2013.

17. Wang K, Degerny C, Xu M and Yang XJ: YAP, TAZ, and Yorkie: A conserved family of signal-responsive transcriptional coregulators in animal development and human disease. Biochem Cell Biol 87: 77-91, 2009.

18. Harvey KF, Zhang X and Thomas DM: The Hippo pathway and human cancer. Nat Rev Cancer 13: 246-257, 2013.

19. Edgar BA: From cell structure to transcription: Hippo forges a new path. Cell 124: 267-273, 2006.

20. Lai D, Ho KC, Hao Y and Yang X: Taxol resistance in breast cancer cells is mediated by the hippo pathway component TAZ and its downstream transcriptional targets Cyr61 and CTGF. Cancer Res 71: 2728-2738, 2011.

21. Huang JM, Nagatomo I, Suzuki E, Mizuno T, Kumagai T, Berezov A, Zhang H, Karlan B, Greene MI and Wang Q: YAP modifies cancer cell sensitivity to EGFR and survivin inhibitors and is negatively regulated by the non-receptor type protein tyrosine phosphatase 14. Oncogene 32: 2220-2229, 2013.

22. Zhang X, George J, Deb S, Degoutin JL, Takano EA, Fox SB, Bowtell DD and Harvey KF; AOCS Study group: The Hippo pathway transcriptional co-activator, YAP, is an ovarian cancer oncogene. Oncogene 30: 2810-2822, 2011.

23. Hall CA, Wang R, Miao J, Oliva E, Shen X, Wheeler T, Hilsenbeck SG, Orsulic S and Goode S: Hippo pathway effector Yap is an ovarian cancer oncogene. Cancer Res 70: 8517-8525, 2010. 